\title{
Charged Higgs Beyond the MSSM at the LHC
}

\section{Katri Huitu*i}

Department of Physics, University of Helsinki

E-mail: katri.huitu@helsinki.fi

\begin{abstract}
The charged Higgs boson is an inevitable particle in supersymmetric models, both in the minimal version and in extensions. It is also a particle, which may have different decay channels depending on the scalar representations in the model, and thus it may help in identifying the model. In this talk I will consider the simplest singlet and triplet extensions of the minimal supersymmetric standard model, and in particular, describe some smoking gun signals of charged Higgs at the LHC collider. Then I will move on to supersymmetric left-right models, in which neutrino masses are naturally generated and which have several dark matter candidates, in addition to possibly solving both the strong and weak CP-problems. I will discuss the charged Higgses, both singly and doubly charged, in such models.
\end{abstract}

Corfu Summer Institute 2016 "School and Workshops on Elementary Particle Physics and Gravity" 31 August - 23 September, 2016

Corfu, Greece

\footnotetext{
*Speaker.

${ }^{\dagger}$ The research presented here has been done in collaborations with P. Bandyopadhyay, S. DiChiara, M. Frank, B. Fuks, D.K. Ghosh, A.S. Keceli, S. Niyogi, S.K. Rai, I. Saha, H. Waltari [1, 2, 3, 4, 5].
} 


\section{Introduction}

A scalar particle, the Higgs boson, found at the LHC in 2012 opened a new era in particle physics. Since then the literature on the Higgs sector and on its extensions has been growing quickly. It is widely thought that the scalar sector may provide hints going beyond the Standard Model, since many of the burning problems in the Standard Model (SM) may be related to the scalar sector: possibly (pseudo)scalar dark matter, generation of neutrino masses using new scalars, generation of fermion mass hierarchies, etc. Supersymmetry is a general framework, where it is natural to have scalars in addition to fermions and gauge bosons in the model describing Nature.

In supersymmetric extensions of the SM, an additional Higgs doublet is needed in order to cancel the anomalies and to generate masses both for up- and down-type quarks. The SM gauge bosons need three scalar degrees of freedom for their longitudinal component, thus leaving five (pseudo)scalar components, which will form five physical Higgs bosons: two neutral scalars $(h, H)$, one neutral pseudoscalar ( $a$ ) and charged Higgs bosons $h^{ \pm}$. Thus any supersymmetric extension of the Standard Model necessarily includes at least one charged Higgs boson.

In this talk, the charged Higgs in extensions of the minimal supersymmetric standard model (MSSM) is discussed. After reviewing the relevant features in the MSSM in view of the mentioned extensions, I discuss a model, in which one singlet is added to the MSSM (NMSSM) [6]. The simplest extension adding a triplet field is the triplet extended supersymmetric model (TESSM), which contains one hypercharge zero triplet in addition to the MSSM representations [7]. These simple models show some essential new features in charged Higgs phenomenology, when new representations are added.

A more complicated situation arises, when one extends the gauge sector to the left-right symmetric one. In supersymmetric left-right models (SUSYLR), one necessarily has richer Higgs sector because of the larger number of groups which need to be broken to the Standard Model gauge group. I will discuss models where the symmetry breaking includes triplets. This is useful since it allows generation of neutrino masses via type-II seesaw mechanism. The used triplets include also doubly charged Higgs bosons, and they are usually the most interesting charged Higgses in such models.

In particular the production and decay modes of the charged Higgs depend on the particular class of models, as well as on mass spectrum. For the purpose of identifying the class of models such features are important.

\section{Charged Higgs in the MSSM}

The prototype of the charged Higgs in supersymmetric models is the MSSM. The charged Higgs in two Higgs doublet model type-2, which also MSSM is, is well studied, see e.g. [8]. In the MSSM the tree-level masses of the Higgs bosons can easily be found from $2 \times 2$-matrices. It is found that if the lightest Higgs is clearly lighter than the others, then the remaining heavy scalar, pseudoscalar and the charged Higgs bosons are nearly degenerate [10].

The decay modes of the charged Higgs can be found and it turns out that irrespective of the ratio of VEVs $\left(v_{2} / v_{1} \equiv \tan \beta\right)$, the charged Higgs decays to $\tau v_{\tau}$ until it is kinematically possible 
to decay to top and bottom. Similarly the favored production channels are found to be through gg fusion and through gb fusion.

The charged Higgs has been searched for at the LHC. The charged Higgs lighter than the top quark was looked for at Run 1. At Run 2 also heavier than top-quark charged Higgs has been serached for in the fermionic decay modes. In the case of Georgi-Machacek -model [9] also $H^{+} \rightarrow W^{+} Z$ was considered. No hints of the charged Higgs has been found, and the mass limit from the studied decay modes have been set to $1-2 \mathrm{TeV}$. If the branching ratios to other channels are significant, the mass limit can be reduced accordingly.

\section{Extensions of MSSM}

The scalar sector of a supersymmetric model can contain other representations in addition to the two doublets considered in the MSSM. A severe constraint on models with additional representations comes from the electroweak $\rho$-parameter, which can be written at the tree-level as [10]

$$
\rho=\frac{m_{W}^{2}}{m_{Z}^{2} \cos ^{2} \theta_{W}}=\frac{\Sigma_{T, Y}\left(4 T(T+1)-Y^{2}\right)\left|v_{T, Y}\right|^{2} c_{T, Y}}{\Sigma_{T, Y} 2^{2}\left|v_{T, Y}\right|^{2}},
$$

where $T$ is the weak isospin, $Y$ is hypercharge, $v_{T, Y}$ is the vacuum expectation value (VEV), and $c_{T, Y}$ is 1 (0) for complex (real) representation. Experimentally $\rho=1.0004_{-0.0004}^{+0.0003}$. For singlets and doublets $\rho=1$ at the tree level, but it constrains models with triplets, if the contributions of various particles do not cancel. Strict cancellation of the triplet VEVs is found in the Georgi-Machacek -model.

\subsection{Singlet extension, NMSSM}

The $\mu$-parameter of the MSSM could be generated without a new scale in the superpotential if a new singlet is added, as happens in NMSSM [6]. In NMSSM the superpotential is

$$
W_{N M S S M}=W_{M S S M}^{\mu=0}+\lambda_{S} S H_{u} \cdot H_{d}+\frac{1}{3} \kappa S^{3},
$$

where the effective $\mu$-term is $\mu_{e f f}=\lambda\langle S\rangle$. There are two new degrees of freedom in the scalar sector of the model, and thus the particle content is three scalars, $h_{i}, i=1,2,3$, two pseudoscalars, $a_{1}, a_{2}$ and the charged scalars $h^{ \pm}$. In NMSSM there are new contributions to the lightest Higgs mass alleviating fine tuning of Higgs mass,

$$
\Delta m_{h}^{2}=\frac{\lambda^{2}}{g^{2}} M_{Z}^{2} \sin ^{2} 2 \beta .
$$

The charged Higgs remains in doublet, and thus it couples similarly to the fermions than the charged Higgs in the MSSM. The lightest particle in the NMSSM Higgs sector can be the lightest pseudoscalar. Such a pseudoscalar has been searched for at the LHC from the decay $h_{1} \rightarrow a a$. This search has not been successful, and we will assume here that $m_{a}>m_{h_{1}} / 2$. The search of the charged Higgs in top decays has been equally unsuccessful, and consequently we assume that a charged Higgs is heavier than the top quark. There is still a possibility that the charged Higgs is only slightly heavier that the top quark, and that the pseudoscalar could be slightly too heavy for 
the on-shell decay of the Higgs boson. Then one could entertain a possibility of the charged Higgs decays to the pseudoscalar and a $\mathrm{W}$ boson [1], which is a distinctly different signal compared to the MSSM charged Higgs decay signals.

Since the charged Higgs couplings to the SM particles is the same as in the MSSM, the production of the charged Higgs in NMSSM is similar than in the MSSM. However, because of the new particles in the Higgs sector, the decay of the charged Higgs is different. It is possible to find benchmark points where

$$
h^{ \pm} \rightarrow a W^{ \pm}
$$

is large or even the dominant decay mode (in our benchmark points the branching ratio varies between $18 \%$ and $66 \%$ ).

We have studied the possibilities for detection in three benchmark points, where the main production channel is $b g \rightarrow t h^{ \pm}$, and the pseudoscalar from the charged Higgs decay decays to taus (BR 10\%) or to the b-quarks (BR 90\%), and the two $\mathrm{W}$ in the process decay either leptonically or hadronically. We found that, if $a \rightarrow \tau \tau$, the increased statistics when one $\mathrm{W}$ decays hadronically is useful in increasing the significance of the signal compared to the background, and for some benchmark points the signal could be seen in early LHC run-2. On the other hand, search with $a \rightarrow b b$ does not seem successful because of large background even if both Ws decay leptonically.

\subsection{Triplet extension, TESSM}

In addition to singlet or doublet extensions, several triplet extensions exist. The simplest possible supersymmetric triplet model can be constructed with a hypercharge zero triplet [7]. Such a representation does not lead to new contributions for the triangle anomaly, and thus it is enough to add a single triplet to the MSSM superpotential:

$$
W_{T E S S M}=W_{M S S M}^{\mu=0}+\lambda H_{d} \cdot T H_{u}+\mu_{D} H_{d} \cdot H_{u}+\mu_{T} \operatorname{Tr}(T T),
$$

where $T$ is a triplet superfield,

$$
T=\left(\begin{array}{cc}
\sqrt{\frac{1}{2}} T^{0} & T_{2}^{+} \\
T_{1}^{-} & -\sqrt{\frac{1}{2}} T^{0}
\end{array}\right),
$$

$\mu_{D}$ is the Higgs doublet mixing parameter and $\mu_{T}$ the Higgs triplet mass parameter. In the triplet there is one new neutral scalar and two new charged scalars. Thus the number of neutral scalars and pseudoscalars is the same than in the NMSSM, while the number of charged scalars triples.

As discussed, the triplet representation restricts the possible VEV of the neutral component due to contribution to the gauge boson masses, and it is found that $\left\langle T^{0}\right\rangle<5 \mathrm{GeV}$. This limit makes it impossible to make the model scale invariant in the same way than the NMSSM, since without $\mu_{D}$ such a small value of the triplet VEV produces a light chargino, excluded by experiments. The experimentally observed Higgs boson mass has new tree-level contributions [11] which alleviates the fine tuning [4]:

$$
\Delta m_{h}^{2}=\frac{2 \lambda^{2}}{g_{2}^{2}+g_{1}^{2}} M_{Z}^{2} \sin ^{2} 2 \beta .
$$

Also the radiative contributions from electroweak sector may be quite large [12]. The new contributions are bigger for smaller $\tan \beta$ and thus our benchmarks have $\tan \beta \sim 5$. One may worry 
about the rare decay $b \rightarrow s \gamma$ for these benchmarks, since there are several new charged Higgses and charginos, contributing to the decay. It turns out that the lightest charged Higgs for our benchmarks is dominantly triplet, and since it does not couple directly to fermions, the contribution to such rare decays is tiny [4].

The qualitatively new coupling in the case of triplets compared to doublets or singlets is treelevel $H^{ \pm} Z W^{\mp}$, which in MSSM is only radiatively generated [13, 14]. In our benchmarks the dominantly triplet charged Higgs has large contributions to the production through this coupling, and the decay of the charged Higgs can have a major branching ratio to $\mathrm{Z}$ and W, see Fig. 1.

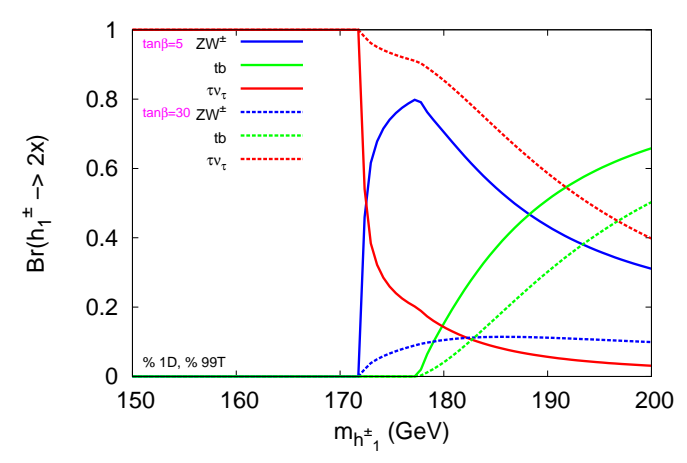

Figure 1: The branching ratios of the lightest charged Higgs for a benchmark scenario [4].

We analyzed in [2] several benchmark points with large triplet contribution in multilepton final states. It seems that three lepton final states can be detected in favourable part of the parameter space, while four to six lepton final states require more than $1000 \mathrm{fb}^{-1}$. Interestingly, since the lightest charged Higgs in TESSM needs not be degenerate with the lightest pseudoscalar or second lightest neutral Higgs - contrary to the MSSM - one can get charged Higgs through $a_{1}$ or $h_{2} \rightarrow$ $h_{1}^{\mp} W^{ \pm}$. This makes it possible to see kinematical edges in the kinematical distributions, from which information also on these heavier Higgs bosons may be deduced [2].

\subsection{Gauge extension, SUSYLR}

A particularly well-motivated model with triplets is the supersymmetric left-right model based on the gauge group $S U(3)_{C} \times S U(2)_{R} \times S U(2)_{L} \times U(1)_{B-L}$, in which the triplets may produce neutrino masses via the type-II seesaw mechanism. Because of the left-right symmetry, the righthanded fermions exist in $S U(2)_{R}$ doublets, making the right-handed neutrino a necessary ingredient in the model. In addition these models have several possible dark matter candidates, see [5], and may possibly solve both the strong and weak CP-problems.

The Higgs sector is rather complicated due to the bigger gauge group than in the Standard Model, and because of the anomaly cancellation requirement. In [5] we considered the following Higgs sector, with which seesaw type-II mechanism is possible and at the same time R-parity is 
conserved:

$$
\begin{array}{rlr}
\Phi_{1}=\left(\begin{array}{cc}
\phi_{1}^{+} & \phi_{1}^{0 \prime} \\
\phi_{1}^{0} & \phi_{1}^{-}
\end{array}\right)=\left(\mathbf{1}, \mathbf{2}, \mathbf{2}^{*}, 0\right), & \Phi_{2}=\left(\begin{array}{cc}
\varphi_{2}^{+} & \varphi_{2}^{0} \\
\varphi_{2}^{0 \prime} & \varphi_{2}^{-}
\end{array}\right)=\left(\mathbf{1}, \mathbf{2}, \mathbf{2}^{*}, 0\right), \\
\Delta_{1 L}=\left(\begin{array}{cc}
\frac{\delta_{L}^{-}}{\sqrt{2}} & \delta_{1 L}^{0} \\
\delta_{1 L}^{--} & -\frac{\delta_{1 L}^{-}}{\sqrt{2}}
\end{array}\right)=(\mathbf{1}, \mathbf{3}, \mathbf{1},-2), & \Delta_{2 L}=\left(\begin{array}{cc}
\frac{\delta_{2 L}^{+}}{\sqrt{2}} & \delta_{2 L}^{++} \\
\delta_{2 L}^{0} & -\frac{\delta_{2 L}^{+}}{\sqrt{2}}
\end{array}\right)=(\mathbf{1}, \mathbf{3}, \mathbf{1}, 2), \\
\Delta_{1 R}=\left(\begin{array}{cc}
\frac{\delta_{1 R}^{-}}{\sqrt{2}} & \delta_{1 R}^{0} \\
\delta_{1 R}^{--} & -\frac{\delta_{1 R}^{-}}{\sqrt{2}}
\end{array}\right)=(\mathbf{1}, \mathbf{1}, \mathbf{3},-2), & \Delta_{2 R}=\left(\begin{array}{cc}
\frac{\delta_{2 R}^{+}}{\sqrt{2}} & \delta_{2 R}^{++} \\
\delta_{2 R}^{0} & -\frac{\delta_{2 R}^{+}}{\sqrt{2}}
\end{array}\right)=(\mathbf{1}, \mathbf{1}, \mathbf{3}, 2), \\
& S=(\mathbf{1}, \mathbf{1}, \mathbf{1}, 0) .
\end{array}
$$

The superpotential of the model is

$$
\begin{aligned}
W= & \left(Q_{L}\right)^{T} Y_{Q}^{1} \Phi_{1}\left(Q_{R}\right)+\left(Q_{L}\right)^{T} Y_{Q}^{2} \Phi_{2}\left(Q_{R}\right)+\left(L_{L}\right)^{T} Y_{L}^{1} \Phi_{1}\left(L_{R}\right)+\left(L_{L}\right)^{T} Y_{L}^{2} \Phi_{2}\left(L_{R}\right) \\
& +\left(L_{L}\right)^{T} Y_{L}^{3} \Delta_{2 L}\left(L_{L}\right)+\left(L_{R}\right)^{T} Y_{L}^{4} \Delta_{1 R}\left(L_{R}\right)+S\left[\lambda_{L} \operatorname{Tr}\left(\Delta_{1 L} \cdot \Delta_{2 L}\right)+\lambda_{R} \operatorname{Tr}\left(\Delta_{1 R} \cdot \Delta_{2 R}\right)\right. \\
& \left.+\lambda_{3} \operatorname{Tr}\left(\Phi_{1}^{T} \tau_{2} \Phi_{2} \tau_{2}\right)+\lambda_{4} \operatorname{Tr}\left(\Phi_{1}^{T} \tau_{2} \Phi_{1} \tau_{2}\right)+\lambda_{5} \operatorname{Tr}\left(\Phi_{2}^{T} \tau_{2} \Phi_{2} \tau_{2}\right)+\lambda_{S} S^{2}+\xi_{F}\right]
\end{aligned}
$$

where generation indices are suppressed for clarity. Following the conventions of Ref. [16], the Yukawa couplings $Y_{Q}^{j}$ and $Y_{L}^{j}$ are $3 \times 3$ matrices in flavour space, the $\lambda$ parameters denote various trilinear Higgs interactions ( $\tau_{2}$ is the second Pauli matrix) and $\xi$ a linear singlet term.

The gauge symmetry is spontaneously broken once the Higgs fields acquire their VEVs,

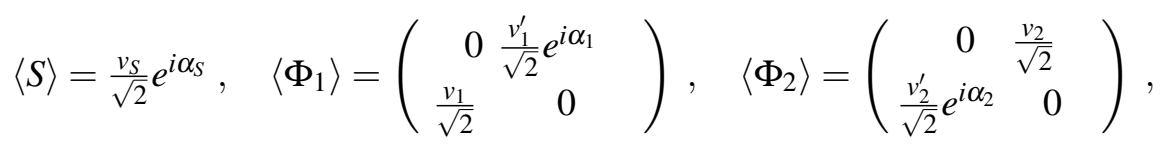

$$
\begin{aligned}
& \left\langle\Delta_{1 R}\right\rangle=\left(\begin{array}{cc}
0 & \frac{v_{1 R}}{\sqrt{2}} \\
0 & 0
\end{array}\right), \quad\left\langle\Delta_{2 R}\right\rangle=\left(\begin{array}{cc}
0 & 0 \\
\frac{v_{2 R}}{\sqrt{2}} & 0
\end{array}\right) \text {, }
\end{aligned}
$$

where the left triplets $\Delta_{1 L}, \Delta_{2 L}$ are assumed to be inert, since they are not needed for symmetry breaking. This is motivated by the constraints arising from the $\rho$ parameter. It is also necessitated by the experimental bounds on the left triplet doubly charged Higgs mass, which can be large enough when originating from the soft supersymmetry breaking mass. The $S U(2)_{R} \times U(1)_{B-L}$ symmetry can be broken by a right-handed triplet. The corresponding VEV needs to be at least at a few $\mathrm{TeV}$ scale, in order to satisfy the experimental limits for the new heavy gauge bosons. The radiative corrections to the right doubly charged Higgs mass must be significant enough to satisfy the current experimental bounds.

The $v_{i R}, v_{1}, v_{2}, v_{1}^{\prime}, v_{2}^{\prime}$ and $v_{S}$ VEVs can be chosen real and non-negative, while the only complex phases which cannot be rotated away by means of suitable gauge transformations and field redefinitions are denoted by the angles $\alpha_{1}, \alpha_{2}$ and $\alpha_{s}$. However, the $C P$-violating $W_{L}^{ \pm}-W_{R}^{ \pm}$ mixing is proportional to $v_{1} v_{1}^{\prime} e^{i \alpha_{1}}$ and $v_{2} v_{2}^{\prime} e^{i \alpha_{2}}$, and is constrained to be small by $K^{0}-\bar{K}^{0}$ mixing data. To reduce the dimensionality of the parameter space, we therefore assume the hierarchy

$$
v_{S}, v_{1 R}, v_{2 R} \gg v_{2}, v_{1}, v_{1}^{\prime}, v_{2}^{\prime} \quad \text { and } \quad v_{1}^{\prime}=v_{2}^{\prime}=\alpha_{1}=\alpha_{2}=\alpha_{S} \approx 0
$$


The upper limit on the tree-level mass of the SM-like Higgs boson can be much larger than in the MSSM by the virtue of the extended gauge sector. If $g_{L}=g_{R}$, one finds [23]

$$
m_{h}^{\text {tree }} \leq \sqrt{2} m_{W} \simeq 113.7 \mathrm{GeV}
$$

a value that can be easily lifted to about $125 \mathrm{GeV}$ by incorporating the radiative corrections and by adjusting the stop masses and mixing. The latter depends on $\tan \beta$, and for values close to one, the tree-level mass of the lightest scalar boson tends to vanish, like in the MSSM. However, the LRSUSY D-terms can increase the tree-level Higgs mass beyond values that are typical from the MSSM.

The second $C P$-even, the lightest $C P$-odd and the lightest singly charged Higgs boson can have masses below or slightly above $1 \mathrm{TeV}$. Close to the alignment limit, their dominant decay modes involve third generation fermions and the related LHC reach is thus similar as for the heavier states of the MSSM. We chose in [5] a moderate value for $\tan \beta$ so that the bounds stemming from both the direct heavy Higgs-boson searches in the $H / A \rightarrow \tau \tau$ channel $[24,25]$ are weaker and the contributions to the rare $B_{s} \rightarrow \mu \mu$ decay are smaller than for large $\tan \beta$. This has an additional advantage to suppress the mixing in the neutral Higgs sector, which may challenge the SM-nature of the lightest state and lead to a large deviation from the SM for the $h \rightarrow b \bar{b}$ branching ratio [3].

In LRSUSY, the doubly charged Higgs sector plays a central role, both in construction of a viable model and in the most useful experimental signals. The doubly charged Higgs mass matrix has a negative eigenvalue at tree-level once the neutral component of the triplet gets a VEV, and one-loop corrections must be included for stabilizing the scalar potential [17]. The original work relied on the lepton-slepton contributions and hence the couplings of the leptons to the right triplet Higgs superfield must be taken large for at least one generation. The same couplings govern the decays of the doubly charged Higgs boson and it is important to verify the consistency with the various LHC bounds. The latter are strong, with the exception of the case in which the doubly charged Higgs boson decays into a ditau final-state [18, 19, 20]. Such searches have set strong bounds on models where the lightest doubly charged Higgs boson is of a left triplet nature. These bounds can be evaded in typical LRSUSY scenarios for right doubly charged Higgs bosons, since the associated production of $\delta^{ \pm \pm} \delta^{\mp}$ through $W_{L}^{ \pm}$is not possible and in the pair production the triplet Higgs couples to the Z-boson only through the $B-L$ and $W_{3 R}$ components leading to a suppression in the production cross section compared to the left-handed triplets.

Whereas one may assume that the triplet Higgs superfield mostly couples to third generation (s)leptons, non-zero couplings to the other generations are needed to generate masses for the righthanded neutrinos. In order to evade all doubly charged Higgs LHC constraints, we fixed in [5] the model free parameters in a way in which the branching ratio of the doubly charged Higgs boson into muons and electrons stays below $10 \%$.

Further constraints arise from the sign of the overall one-loop correction to the doubly charged Higgs mass, which depends on the slepton masses and $v_{R}$. If the slepton masses are much smaller than $v_{R}$, the correction will be negative and worsen the problem of the negative mass eigenvalue [21]. Recently it was found that the gauge and Higgs sectors can also significantly contribute to the doubly charged Higgs mass, which opens up a window for lighter right sleptons and sneutrinos [22]. To obtain a heavy enough doubly charged Higgs boson, we set the $\lambda_{R}$ parameter to a 
large value, which leads to a large contribution to the doubly charged Higgs boson mass from the Higgs sector [5].

Although in [22] the largest values for $m_{\Delta^{ \pm \pm}}$were around $650 \mathrm{GeV}$, stable vacua can still be achieved with doubly charged Higgs boson masses slightly above $800 \mathrm{GeV}$. The doubly charged Higgs boson decaying to the tau leptons is less constrained than if the decay would be to electrons and muons, due to the efficiency of tau identification. Excluding a $800 \mathrm{GeV}$ doubly charged Higgs boson decaying solely to same-sign taus would require an improvement of two orders of magnitude with respect to the latest CMS bound [20], which will be challenging even with $3000 \mathrm{fb}^{-1}$. It is therefore uncertain whether the LHC will be able to exclude the model on the basis of doubly charged Higgs boson searches only due to the structure of the Yukawa couplings (and the various doubly charged Higgs boson branching ratios). If the vacuum is stabilized by the introduction of $B-L=0$ triplets, the doubly charged Higgs boson can be heavier and outside the reach of the LHC.

Turning to singly charged Higgs bosons, indirect constraints originating from $b \rightarrow s \gamma$ data [26] suggest that they must be heavy [27], at least if there are no cancellations in the SUSY loopcontributions to the singly charged Higgs boson mass. This can be accommodated in LRSUSY setups if $\tan \beta_{R}=v_{2 R} / v_{1 R}$ deviates from one and if $v_{R}$ is large. Such a deviation will decrease one of the diagonal elements of the doubly charged Higgs mass matrix, and subsequently make it more difficult to satisfy the doubly charged Higgs mass constraints. We therefore adopt

$$
\tan \beta_{R} \simeq 1.05,
$$

which, with the values for $v_{R}$ used in [5], pushes all the MSSM-like Higgs states to be heavier than current LHC bounds. They have masses squared proportional to $g_{R}^{2} v_{R}^{2}\left(\tan ^{2} \beta_{R}-1\right)$ and are hence at most just above the $\mathrm{TeV}$ scale. Moreover, all additional scalar bosons have masses of the order of $v_{R}, v_{S}$, or of the left triplet soft mass parameters and hence are a lot heavier.

In addition to the signals due to the doubly charged Higgs boson, interesting signals of the supersymmetric left-right model can be those mediated by the right-handed gauge boson $W_{R}$, as studied in [5].

\section{Summary}

The singlet and triplet extensions of MSSM can lead to alleviated fine tuning of the Higgs mass. In the discussed extensions interesting new signals for the charged Higgs can be found. The discussed charged Higgs decay to a pseudoscalar and $W$ cannot happen in MSSM. Similarly processes originating from tree-level $H^{ \pm} W^{\mp} Z$ are not present in MSSM or in NMSSM.

Another discussed extension, including extension of the gauge symmetry, is the supersymmetric left-right model, in which many current problems of particle physics can be solved. The Higgs sector of the model becomes quite large. In addition to the previous signals, the SUSYLR model Higgs sector has new interesting features. In particular, there is a relatively light doubly charged Higgs boson, which may decay dominantly to the tau leptons.

The discovery of a doubly charged Higgs boson would not be a signal specific to LRSUSY setups and should be used in conjunction with other measurements to draw conclusive LRSUSY statements. The discovery of a doubly charged scalar field along with doubly charged higgsinos and 
a right gauge boson would be a strong hint towards establishing a left-right supersymmetry without discovering any other SUSY particle, simply by virtue of the robustness of the signal. Such signals have therefore been studied widely in the literature.

\section{References}

[1] P. Bandyopadhyay, K. Huitu and S. Niyogi, "Non-standard charged Higgs decay at the LHC in Next-to-Minimal Supersymmetric Standard Model,” JHEP 1607 (2016) 015 [arXiv:1512.09241 [hep-ph]].

[2] P. Bandyopadhyay, K. Huitu and A. Sabanci Keceli, "Multi-Lepton Signatures of the Triplet Like Charged Higgs at the LHC," JHEP 1505 (2015) 026 [arXiv:1412.7359 [hep-ph]].

[3] M. Frank, D. K. Ghosh, K. Huitu, S. K. Rai, I. Saha and H. Waltari, "Left-right supersymmetry after the Higgs boson discovery," Phys. Rev. D 90 (2014) no.11, 115021 [arXiv:1408.2423 [hep-ph]].

[4] P. Bandyopadhyay, S. Di Chiara, K. Huitu and A. S. Keceli, "Naturality vs perturbativity, Bs physics, and LHC data in triplet extension of MSSM," JHEP 1411 (2014) 062 [arXiv:1407.4836 [hep-ph]].

[5] M. Frank, B. Fuks, K. Huitu, S. K. Rai and H. Waltari, "Resonant slepton production and right sneutrino dark matter in left-right supersymmetry," arXiv:1702.02112 [hep-ph], to be published in JHEP.

[6] U. Ellwanger, C. Hugonie and A. M. Teixeira, "The Next-to-Minimal Supersymmetric Standard Model," Phys. Rept. 496 (2010) 1 [arXiv:0910.1785 [hep-ph]].

[7] J. R. Espinosa and M. Quiros, "Higgs triplets in the supersymmetric standard model," Nucl. Phys. B 384 (1992) 113.

[8] A. G. Akeroyd et al., "Prospects for charged Higgs searches at the LHC," arXiv:1607.01320 [hep-ph].

[9] H. Georgi and M. Machacek, Nucl. Phys. B 262 (1985) 463.

[10] J. F. Gunion, H. E. Haber, G. L. Kane and S. Dawson, “The Higgs Hunter's Guide," Front. Phys. 80 (2000) 1.

[11] S. Di Chiara and K. Hsieh, “Triplet Extended Supersymmetric Standard Model," Phys. Rev. D 78 (2008) 055016 [arXiv:0805.2623 [hep-ph]].

[12] P. Bandyopadhyay, K. Huitu and A. Sabanci, "Status of $Y=0$ Triplet Higgs with supersymmetry in the light of $125 \mathrm{GeV}$ Higgs discovery," JHEP 1310 (2013) 091 [arXiv:1306.4530 [hep-ph]].

[13] J. F. Gunion, G. L. Kane and J. Wudka, "Search Techniques for Charged and Neutral Intermediate Mass Higgs Bosons,” Nucl. Phys. B 299 (1988) 231.

[14] A. Mendez and A. Pomarol, "One loop induced $H^{+} W^{+} Z$ vertex in the minimal supersymmetry model,” Nucl. Phys. B 349 (1991) 369.

[15] K. Huitu and J. Maalampi, "The Higgs sector of a supersymmetric left-right model," Phys. Lett. B 344 (1995) 217 [hep-ph/9410342].

[16] A. Alloul, M. Frank, B. Fuks and M. Rausch de Traubenberg, "Chargino and neutralino production at the Large Hadron Collider in left-right supersymmetric models," JHEP 1310 (2013) 033 [arXiv:1307.5073 [hep-ph]].

[17] K. S. Babu and R. N. Mohapatra, "Minimal Supersymmetric Left-Right Model," Phys. Lett. B 668, 404 (2008); 
[18] S. Chatrchyan et al. [CMS Collaboration], "A search for a doubly-charged Higgs boson in $p p$ collisions at $\sqrt{s}=7$ TeV,” Eur. Phys. J. C 72 (2012) 2189 [arXiv:1207.2666 [hep-ex]].

[19] CMS Collaboration [CMS Collaboration], "Search for a doubly-charged Higgs boson with $\sqrt{s}=8 \mathrm{TeV} p p$ collisions at the CMS experiment," CMS-PAS-HIG-14-039.

[20] CMS Collaboration [CMS Collaboration], CMS-PAS-HIG-16-036.

[21] K. S. Babu and A. Patra, Phys. Rev. D 93 (2016) no.5, 055030 [arXiv:1412.8714 [hep-ph]].

[22] L. Basso, B. Fuks, M. E. Krauss and W. Porod, "Doubly-charged Higgs and vacuum stability in left-right supersymmetry,” JHEP 1507 (2015) 147 [arXiv:1503.08211 [hep-ph]].

[23] K. S. Babu, X. G. He and E. Ma, "New Supersymmetric Left-Right Gauge Model: Higgs Boson Structure and Neutral Current Analysis,” Phys. Rev. D 36 (1987) 878.

[24] V. Khachatryan et al. [CMS Collaboration], "Search for neutral MSSM Higgs bosons decaying to a pair of tau leptons in pp collisions," JHEP 1410 (2014) 160 [arXiv:1408.3316 [hep-ex]].

[25] M. Aaboud et al. [ATLAS Collaboration], "Search for Minimal Supersymmetric Standard Model Higgs bosons $H / A$ and for a $Z^{\prime}$ boson in the $\tau \tau$ final state produced in $p p$ collisions at $\sqrt{s}=13 \mathrm{TeV}$ with the ATLAS Detector," arXiv:1608.00890 [hep-ex].

[26] T. Saito et al. [Belle Collaboration], "Measurement of the $\bar{B} \rightarrow X_{s} \gamma$ Branching Fraction with a Sum of Exclusive Decays,” Phys. Rev. D 91 (2015) no.5, 052004 [arXiv:1411.7198 [hep-ex]].

[27] T. Hermann, M. Misiak and M. Steinhauser, " $\bar{B} \rightarrow X_{s} \gamma$ in the Two Higgs Doublet Model up to Next-to-Next-to-Leading Order in QCD,” JHEP 1211 (2012) 036 [arXiv:1208.2788 [hep-ph]]. 